\title{
Interactive Media Art and Design in the Sensory Experiences
}

\author{
Ting Xu \\ Academy of Art and Design, Dalian Polytechnic University, China
}

Copyright $\subseteq 2019$ by authors, all rights reserved. Authors agree that this article remains permanently open access under the terms of the Creative Commons Attribution License 4.0 International License

\begin{abstract}
Nowadays, people in the era of Internet could experience both real and virtual spaces. Along with the progressive diversification, the definition of design has extended due to the application of multi-media. Interactive media art design, an emerging concept beyond traditional media, not only combines the application of many media, but also highlights the complementarity of electro-optic technology and interactive design. This is interactive media art design, which is interdisciplinary and diversified. Why research interactive media art design? Because it is different from the traditional art design form. Interactive media art and design includes not only interface design, but also such interactive design as motion sensing, virtual reality, holographic images and other means yet to be developed. Media and human senses are linked in some way. To some extent, media is sensory extension. Hence, it is important to sensory perception experience. Interaction exists in our daily life, which consists of interaction of human beings and things as well as the Internet of things. Therefore, the synchronization of art design, sciences and age has to be considered. Interactive art and design is from the life and for the life. It breaks the unidirectionality conveyed to people by previous works, the subjective transmission of works to experiencers. Moreover, it breaks the traditional design concept of designers. The interactivity makes the work humanized, so that people have a stronger participation reflected in it, and thus has a more vivid experience. In interactive design, the sensory experience is produced by the experiencer, which strengthens the emotion experience. In the interactive art design experience, a tangible or intangible "interface point", the beginning of sensory experience, has been created between people and objects, surface and surface. A full range of perception including sight, hearing, and touch has been set up, while time and space need to be considered. To better experience interactive design, the knowledge of interaction design and its significance shall be understood. Only in this way can designers better expand interaction design and make participators have more experiences. The sensory experience in interactive design is endowed with
\end{abstract}

more humanity, which shows a new form of art design emerging in creative industry. The development of interactive design will lead a new trend of art design, and is bound to create an industrial chain and enhance the development of other industries. The interactive media art and design must consider the participators, and have them fully involved and experienced. Interactive design will gradually make our life more colorful.

Keywords Electro-optic Era, Interactive Media Art and Design, Sensory Experiences, Interface Point

\section{The Overview of Interactive Media Art Design}

\subsection{Interactive Media Art Design Background}

Nowadays, people living in the Internet age are in the space of reality and virtuality. In this era of pluralism, the use of many media has broadened the definition of design, and a new force has gradually grown and expanded. The medium not only combines a variety of media forms, but also the use of interactive design in the current era of electricity and light is more complementary. This is interactive media art design, which is an interdisciplinary design and a diversified design.

\subsection{Why Research Interactive Media Art Design}

This topic is different from the traditional art design, which focuses on the one-way information transmission. Works or products are presented to the viewer, who only needs to watch passively while the listener listens passively. In interactive art design, viewers and listeners can participate in it as participants to experience, and information transmission turns into two-way. This is also its most distinctive feature. 


\subsection{The Definition of Interactive Media Art Design}

Different researchers in the world have different expressions. For example, Myron Kruger (1983) mentioned Artificial Reality, Paul Milgram, Fuio Kishino (1994) and Ginatatus, V (2007) referred to the mix reality ${ }^{1}$. Roy $\operatorname{Ascott}^{2}$ (2002) argued technoetic art. Stephen Wilson (2002) said information art, namely, such as robot, The development of artificial life, gesture, touch, etc. Jay David Bolter and Diane Gromala (2004) mentioned that digital art is practiced by graphic artists who use traditional video and audio as well as computers to create art installations and exhibits. Their works are usually commercial or popular. Levin Golan (2006) and Woolner, A (2009) showed interactive art in their article, and mainly explained Computer vision. Wolf Lieser (2010) indicated the digital art, namely interactive objects and art in public space. Cynthia Breazeal (2010) noted the blended reality.

Different researchers in China have different ideas, such as the multimedia and network art indicated by Wan Yan, Wan Rong and Chen Shaohua (2007), and the multimedia art design mentioned by Hu Jie and Yan Chen (2009). The main structure of the interface. Wang Feng and Ma Hui (2009) argued multimedia technology. Mainly explain software such as flash animation design and production. Liu Shuming, Hou Yue (2014) showed the interactive element design of digital media. In this paper, the design of interactive interface is described, which defines interactive elements including text, icon, navigation bar, text box and so on. In the previous research, different researchers have different focuses, but they are either inclined to art or technology, and the content is not comprehensive enough. I extracted their core concepts, and then thought and summarized the concept of "interactive media art design".

Interactive media art design refers to the interlaced and influential art design forms presented on various media. It is a new media art design centered on interactive ideas and interactive technologies ${ }^{3}$. Interactive media art design is a combination of modern information technology and art design forms such as electronic technology, multi-media technology, sensor technology and detection technology. It is the realization process of designers using modern science and technology expression.

Interactive media art design is reflected in many fields. It uses a variety of media forms to extend the definition of space art, performance art, graphic design, and digital media art. In the field of art design, such as media art, space art, product design, through the interaction between people

1 Mix reality, sometimes referred to as hybrid reality, is the merging of real and virtual worlds to produce new environments and visualizations where physical and digital objects co-exist and interact in real time. 2 Roy Ascott works with cybernetics and telematics, on an art which is technoetic, focusing on the impact of digital and telecommunications networks on consciousness. Built a theoretical framework for approaching interactive artworks.

3 New media art design: mainly based on "optics" media and "electronic" media are basic languages, and an interactive and comprehensive art design based on digital technology. and works, there are a variety of artistic design creations where the senses may coexist.

This video art installation brings people visual and psychological feelings through different video and installation spaces inside.

Virtual reality works bring different experiences to people, and holographic images give people a visual psychological experience. The interactive art of space gives people different feelings through images or pictures played in the environment (Figure 1). The interface design (Figure 2), including the mobile interface, web interface, game interface and other interfaces, gives a visual, tactile, and auditory experience. The somatosensory interaction has more physical experience, and there are other interactivity designs that have yet to be developed.

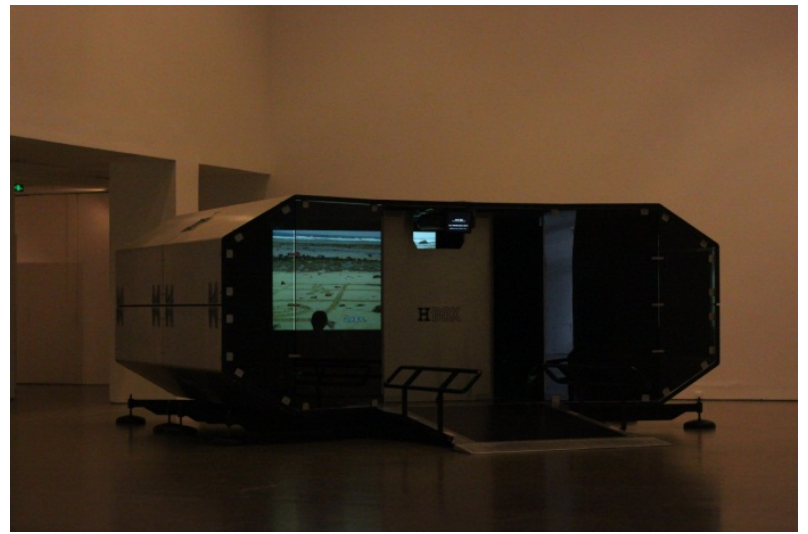

Figure 1. X-BOX, Xu Ting, 2012

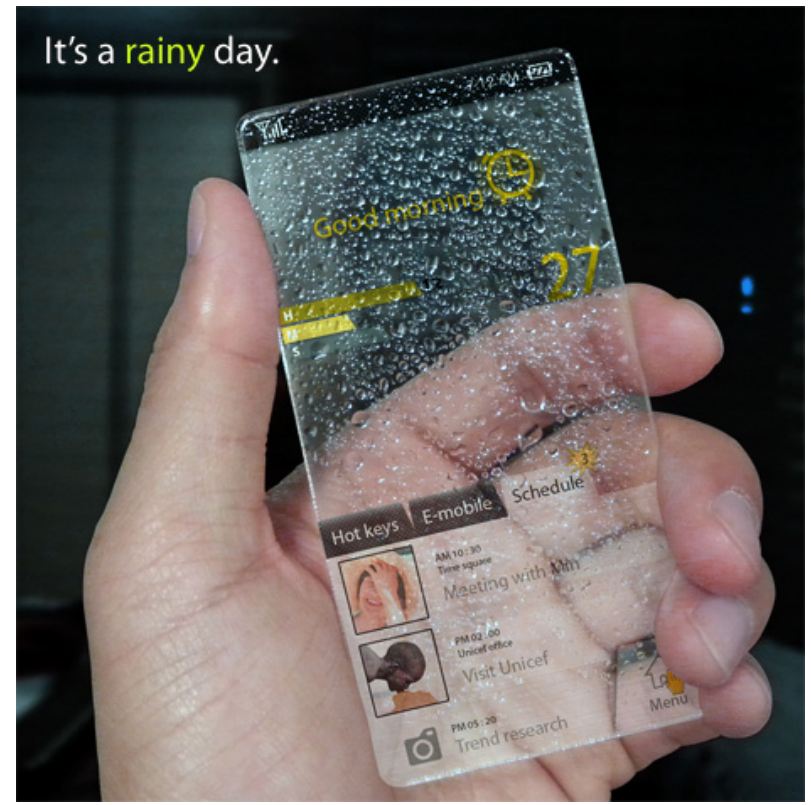

Figure 2. Interface design, Xu Ting, 2012

\section{The Interactive Media Art Design in the Age of Electricity and Light}

Interactive design mainly exists in the era of electricity 
and the future with the light form as the leading era. Nowadays, the network that we cannot do without one day needs to rely on electricity to provide energy. In the city, it can be said that the degree of development of power has symbolized the development of a city to some extent. It is not difficult to see that the development of a city, its night is also brightly lit, so electricity as a medium in life, we cannot do without it.

From the initial use of electric lights to the current home appliances become a must, from the spread of radio and television to the widespread use of the Internet, the medium of the power era has its own unique art form. With the electricity, the media carries out the transmission of language, vision, hearing and other sensory arts, such as radio communication, TV commercials, stage art design, interactive media art design, etc. Among them, interactive media art design has greater development potential in life and market.

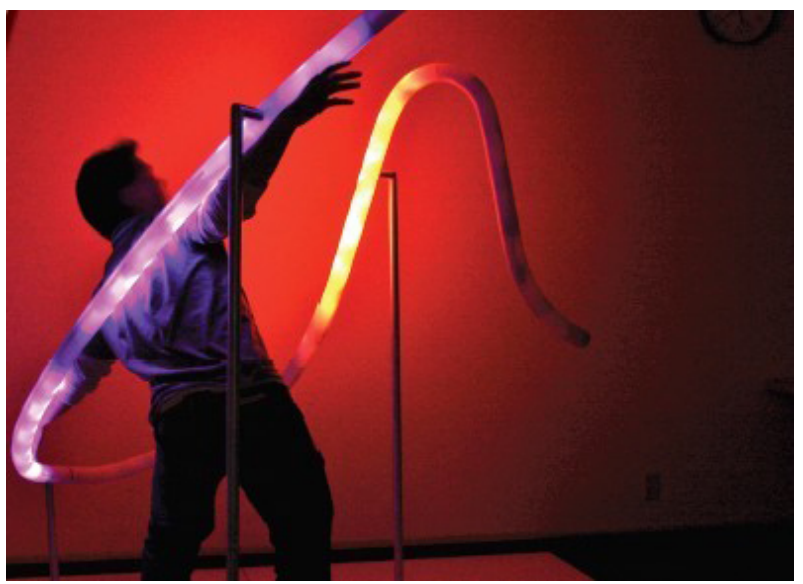

Figure 3. Massachusetts institute of technology, team work, Xu Ting, 2012

With the development of electricity, the application of light will gradually occupy a dominant position in the future. With the development of science and technology, human innovation has gradually improved, and the use of light energy has also entered an unprecedented transcendence in the future. Artistic design and creation integrates all kinds of media into it.

\section{The Sensory Experience of Interactive Media Art Design}

\subsection{Media and Sensory Experience}

Interactive design of a variety of media forms at Herbert Marshall McLuhan(1964), the definition of the medium is more extensive, the medium is everything, the medium is closely related to the human sense, all the media can have some connection with the human senses, the sense refers to the animal feeling the external stimulus organ. Such as the ear, eyes, tongue, nose, skin and so on.
For example, the stone axe is the extension of the hand, the wheel is the extension of the foot, the book is the extension of the eye, the broadcast is the extension of the ear, the clothes are the extension of the skin, and the pinyin text is a visual extension... The media is everywhere. Everything that can make people or people, people and things or things and things have a relationship is a broad medium, and these are all interactions all the time, so the medium used in artistic creation can be very extensive, it can break our traditional sense of paper media and more different media creative use. To some extent, the media is an extension of the senses.

The perception experience of sensory is especially important. Perception is the collective term for feeling and consciousness. Our personal perception is not a closed system, but constantly transforming into experience. We call this experience consciousness. But our extended perceptions, tools, and techniques have been closed systems since ancient times, and they cannot interact, nor can they enter the collective consciousness of human beings. Nowadays, in the era of electricity, the nature of our technical tools to instantly coexist with information has caused new changes in human history. Our extended faculty and perception constitute a unified field of experience that requires our faculty and perception to become collectively conscious. Just like our personal senses, our technology needs to have a mutual effect and a certain ratio in order to make rational coexistence possible. As long as our technology moves slowly like wheels, alphabets or currencies, they are socially and psychologically tolerable as separate, closed systems. But this is no longer the case. Today's video, sound and motion are on a global scale. At the group level, the ratio of interactions in the extension of human faculties becomes a necessity for the collective, just as individual rationality has always been necessary ${ }^{4}$.

\subsection{The Multi-sensory Communication Based on Vision}

However, in the five senses of sight, touch, taste, hearing and smell, visual design is particularly important, including why interactive art design belongs to visual communication, and it has certain truth. Visual communication can be aided by tactile communication, but tactile communication cannot be reversed by visual communication. It can only gradually explore the shape of things through direct contact, and the vision can be seen at a glance. Although the sound in the auditory communication is easy to approach in complex space and time, the auditory communication has limitations. For example, a bird is sing, its song is only generated at that moment, and the sound is gone after the past. And through visual communication we can preserve it at the moment of

4 The individual rationality past is called the individual perception. 
the song. In contrast, the advantages of visual communication are revealed. It is not only a high-definition medium, but also provides information about various objects and events in the external world. For example, in visual communication, bird singing, bird image and color will be presented to the audience as complete and clear as possible.

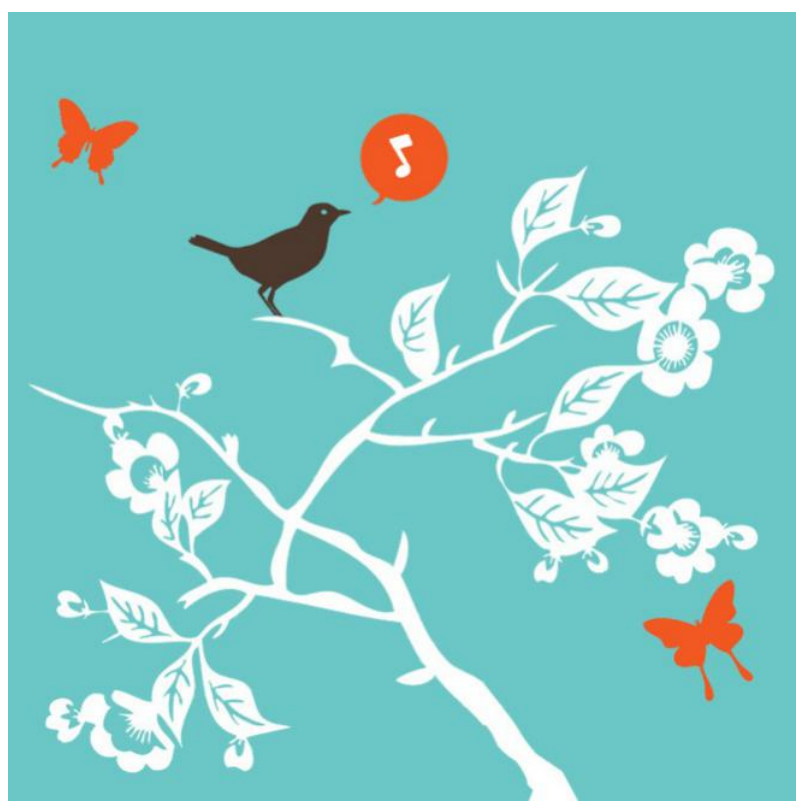

Figure 4. Birds are singing, $\mathrm{Xu}$ Ting, 2012

As early as before, McLuhan put forward a very profound insight into the senses. He believes that any culture is an orderly organization with sensory bias. In tribal societies, for very practical reasons, touch, taste, hearing, and smell are very developed, it's more developed than strictly visual. Suddenly, the phonetic alphabet landed in a tribal society like a bomb, putting the vision at the highest level of the sensory system. Literacy introduces people to tribal societies, allowing them to replace their ears with their eyes, linear visual value and sense of division replace the whole, profound and public interaction. Phonetic alphabet is the enhancement and enlargement of visual function. It weakens the functions of hearing, touch, taste and smell, penetrates into the discontinuous culture of tribe people, and transforms his organic harmony and complex synaesthesia into the way of the consistent, continuous and visual perception. He believes that the phonetic alphabet is fundamentally different from the ancient and rich holy text and hieroglyphics. Egypt, Babylon, Maya and Chinese culture are indeed an extension of the senses. But this extension is an extension of the following meaning: they use graphics to represent reality, and they use many symbols to cover a wide range. This is very different from phonetic alphabet. Phonetic alphabet meaningless letters are corresponding to meaningless languages. It can contain all meanings and all languages in a small number of letters. This achievement requires that the forms and sounds of words be separated from their linguistic and dramatic meanings in order to make the actual sounds visible. It forces visual function away from its interaction with other senses. This leads to its rejection of consciousness in the crucial areas of our perceptual experience, which in turn leads to unconscious atrophy. The balance of the sensory system -- or the gestalt of sensory interaction and the psychological and social harmony that comes out of that balance -- needs to be broken. The visual function is overdeveloped. In the other words, there is no such situation. At this point, the visual sense in a sense appears to be more important location. The externalization and occurrence of perception is language or speech, which is a tool to "enable people to accumulate experience and knowledge with a form, which makes the transmission and maximum utilization of information possible. (Leslie Alvin White, 1949, p240)

In the experience that gives priority to with visual sense, interactive it is the topic that designs eternity. Dan Boyarski, former dean of the school of design at Carnegie Mellon University, once said that the goal of interaction is the user experience, the user has a good experience, and the designer's experience is integrated with the human experience. Interaction is about communication. $\mathrm{Lu}$ Xiaobo, director of the information art design department of the academy of fine arts at Tsinghua University, said that interactive art design enhances and expands people's life, work, study and communication by creating user experience.

\subsection{The Time and Space in the Experience}

Interaction has irreplaceable function in the media, in the media art design, a wide range of what we call the interaction, the experience is very wide, including the people communicate with each other, people have a common goal, communication between human and space (a person from one space to another space), human and network, object and object belong different experience among the interaction.

"We need to design for experience, design for space, design for time, design for real interaction," says Paul Neervoort, creative director of interactive design at Philips quality life. Explain the method of change, design for experience, what customers need, what kind of design is needed, if from the customer's point of view, will consider is for entertainment, or for efficiency? If malls are all for customer needs then distinction, is there a different mall? Some malls are designed according to customers' needs. Elevators, escalators, people, coffee places, etc., are all prepared for improving user experience. All customer needs should be considered.

There are four aspects of user experience to consider:

1. Time: use time, let time for our service, for the time design, how to make full use of time and save time better browsing. 
2. Space: understand the space, and feel the space with audio-visual and body. For example, if we design for the table, how can we put the conference things on the table, do not think about the place, can subconsciously go to a certain position, so as to achieve something, strengthen the experience of a certain space, software and space interface is the same, how to grasp this experience. For example, when we see a room, we can remember the space and find what we want, and our space is composed of many interfaces.

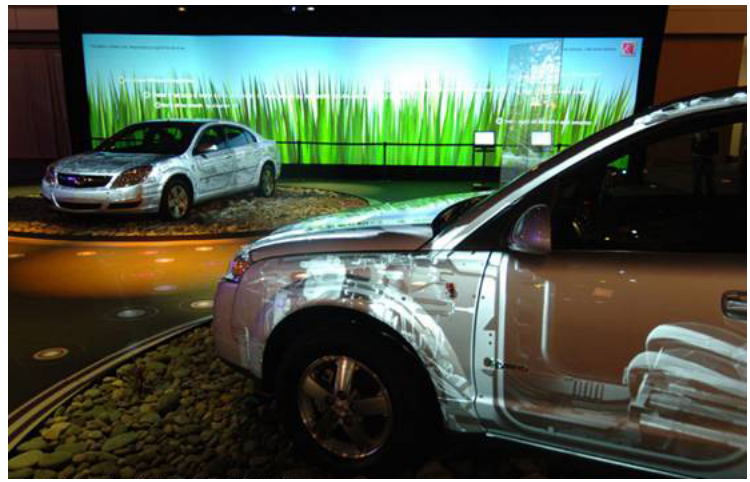

Figure 5. Grass Interactive installation design at the show, $\mathrm{Xu}$ Ting, 2012

\section{The Bridge to Perceive the World -- the Interface Point}

The bridge between the media and the perceived world is the "interface point". The media exists in our life and affects people. We need to act and survive, and we cannot do without the media at any time. The point of contact formed by the tangible or intangible interface between the human senses and various works is called the "interface point"(Xu Ting, 2012, p52, p53). This is also a relatively common creation method of interactive media art, since people are the subject of appreciating works and have the characteristics of subjective initiative; there are many interactive creations that take people as the trigger point of works. Interactive media art is characterized by juxtaposing multiple senses into a work. Human senses, such as hands, feet and ears, come into contact with the work or operate at a distance, and interact with various tangible or intangible interfaces. It is also one of the common techniques of expression in interactive media art.

Tangible and intangible interfaces include web browsing interface, computer system and software operation interface, cable TV interactive on-demand interface, mobile communication product interface, electronic product interface. From the perspective of technology, the interface can be divided into screen interaction interface, touch interaction interface, voice control interface, image interaction interface, perception interaction interface, $\mathrm{AR} / \mathrm{VR}$ interaction interface, automatic recognition interaction interface, robot interaction interface, media interaction interface, integrated media interaction interface (Guo Xiao dong \& He Yujin, 2008). Various artistic design works can be developed by using different interfaces. The touch interface of the mobile phone can form a tangible interface point between the person and the face, the network interface mouse and the network to form a interface point, the game people and the computer to form a interface point, and the motion of the interaction between the people and participate in the game invisible interface point. All kinds of interesting experiences are waiting to be developed to enrich our life.
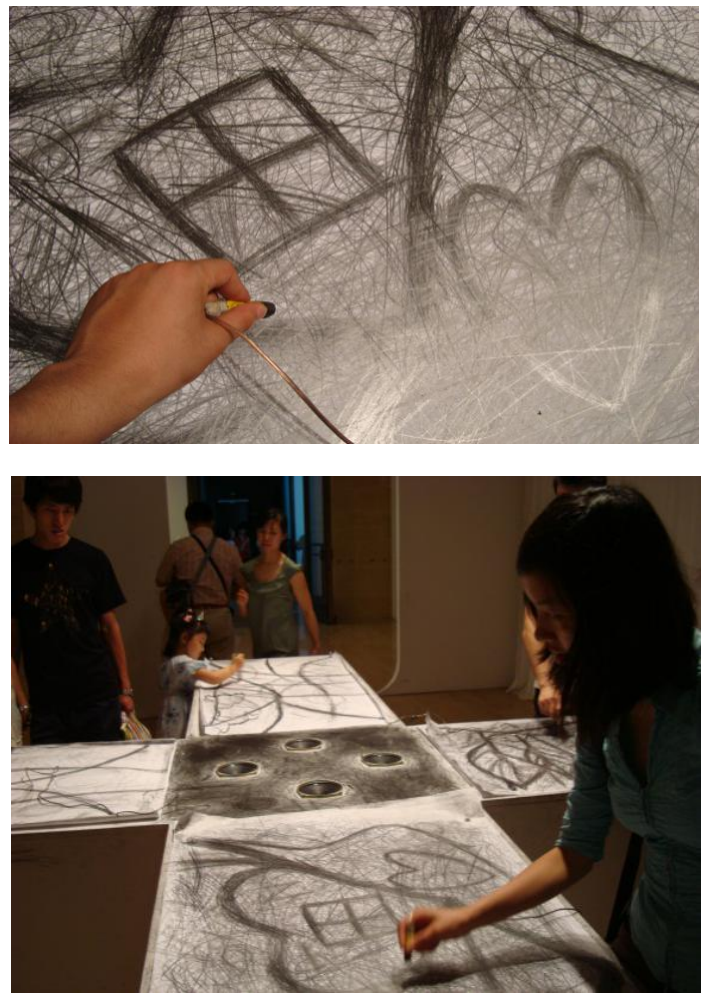

Figure 6. Jin Jizhe, Sound painting (Korea) 2001-2008, Xu Ting, 2012

In this case, when the experiencer is drawing with a "pen", the work will produce different sounds according to the strength, way or stroke of the experiencer.

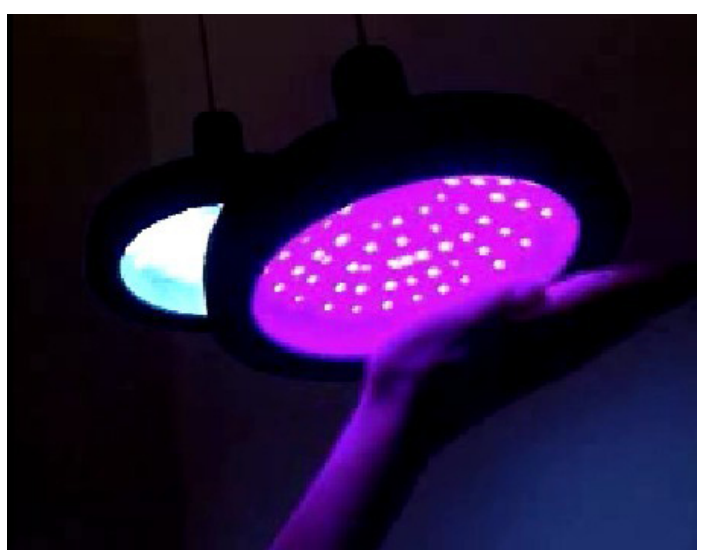

Figure7. Sense light, Xu Ting, 2012 
In 2006, Philips launched an interactive product. With the shaking of people's hands in front of the light, the strength of the light is constantly changing.

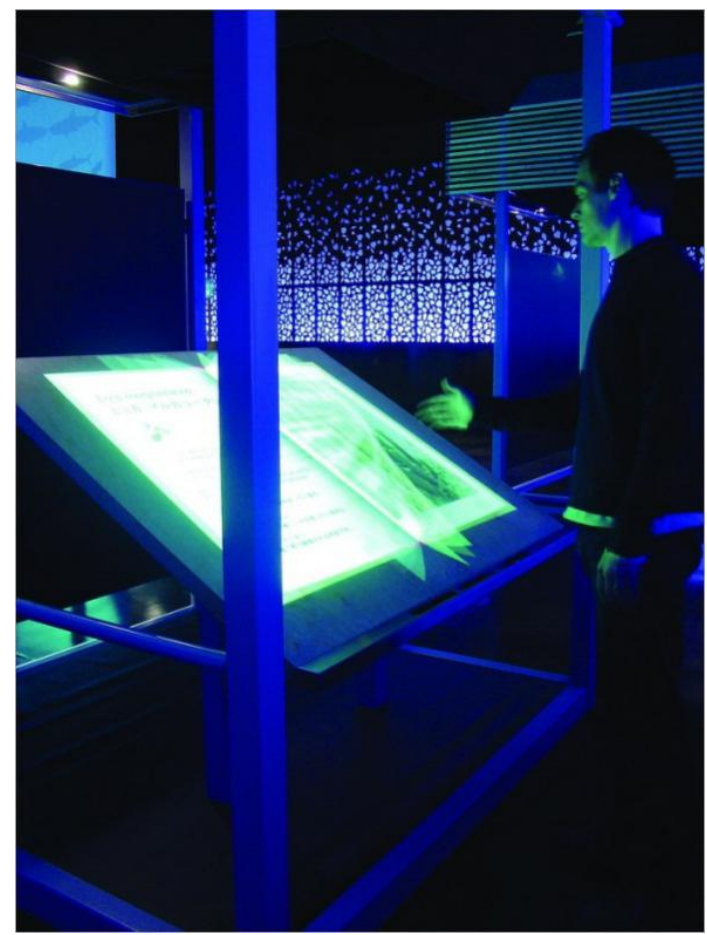

Figure 8. Intangible Interface, Xu Ting, 2012

\section{The Outlook of Interactive Media Art Design}

\subsection{The Embodiment of Interactive Media Art Design in Life}

With the development of the information age, the Internet has connected people all over the world. People have more interactions through the Internet. From the previous traditional media, radio, television and other media all affect our auditory, visual and other senses, including mobile phones as a convenient media. Make us feel a good-looking, fun, different tastes and even feel delicious gluttonous feast is reflected in a medium.

Now the popularity of the network and the depth of influence, the development of science and technology, human sensory experience is even more pervasive. According to the principle of "interface point", we can touch the screen and feel the birdsong and fragrance of flowers simulated by the machine. The interactive design makes our senses have a more comprehensive and interesting experience.

There are also many examples of interactive media art application in daily life, such as some shopping malls or tourist resorts, which are equipped with glass covers on the ground, and there are animations of fish in them. Where tourists go, the small fish all run away, enhancing the fun of tourists through interactive art.

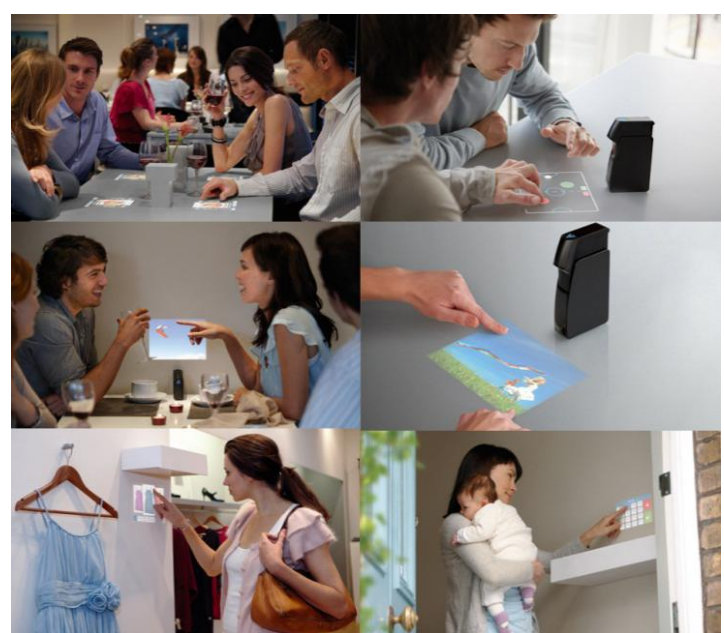

Figure 9. The Holographic Projector, Xu Ting, 2012

High-resolution pictures and images can be projected onto any surface, and the user can then control the projected content through gestures. After the content is projected onto the object surface, the projector will sense the finger movement of the operator through the infrared motion sensor, so as to perceive the intention of the operator and adjust the broadcast content accordingly.

Interaction design has produced people with work experience, with the development of the Internet of things, by means of radio frequency identification (RFID), infrared sensors, global positioning system (GPS), such as the laser scanner information sensing device, according to the contract agreement, to any object connected to the Internet, information exchange and communication, to achieve the object of the intelligent identification, location, tracking and monitoring and management of a network (Alan Dix et al., 2006).

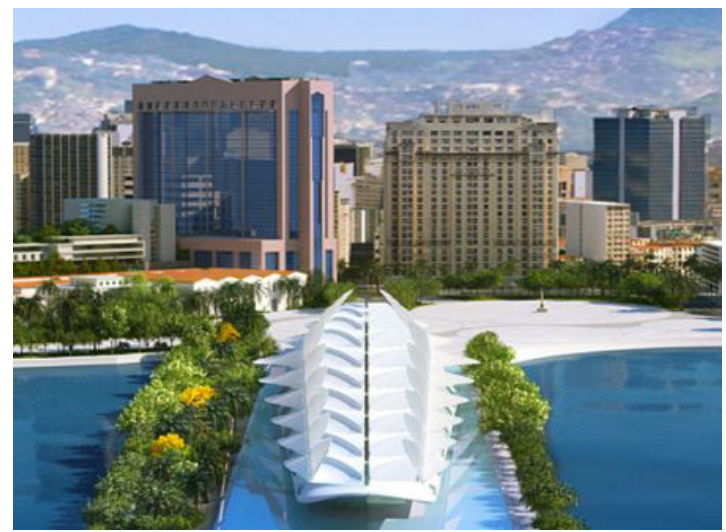

Figure 10. Rio DE Janeiro Museum, Xu Ting, 2012

Located in Rio DE janeiro, Brazil, the museum was designed by Santiago calatrava, a famous architect. It is one of a series of urban renewal projects for the 2016 Olympic Games in Rio DE janeiro. The exterior of the museum is cantilevered, and the roof panels, arranged like outstretched sails, can be angled to suit the changing environment. 


\subsection{The Outlook of Interactive Media Art Design}

The sensory experience in interactive design makes the design more humanized. It is an embodiment of the emerging art design form in the creative industry. Its perfection and development is bound to be a trend of the future development of art design. It is also bound to produce the relevant industrial chain and influence the development of other industries.

Interactive media art design is the combination of media art design and science; all kinds of multi-sensory technologies are indispensable. Therefore, human factors should be taken into consideration when making interactive design, allow people to fully participate in and experience, interactive design will be the human sensory experience the release of the accelerator, and it gradually perfect will make our life more colorful.

\section{Acknowledgement}

This paper, as an important part of the book interactive media art, the second edition is out now. Sincerely thanks professor Liu Guanzhong, professor Ren Jian, professor Song Xiewei, President Xin Xiangyang, President Wang Shouping, and chief editor Cang Xiaodong for their support and help in making this book serve as the textbook of "the 12th five-year plan" national art design experimental teaching demonstration center! This textbook has been approved as a national teaching material, and I would like to thank Tsinghua university, China designers association and other universities and institutions for their attention to interactive media art design!

\section{REFERENCES}

[1] Alan Dix, Gregory d.arbowd, Janet Finlay, Runsell Beale. Human-coumputer Interaction. Beijing: Electronic industry press. 2006

[2] Marshall McLuhan. Understanding Media: The Extensions of Man. Cambridge, Massachusetts: MIT Press. 1964.

[3] Leslie Alvin White, Culture of Science: A study of man and civilization, pp240.1949.

[4] $\mathrm{Xu}$ Ting. Interactive Media Art. Shenyang: Liaoning province fine arts publishing house. 2012.

[5] Guo Xiaodong \& $\mathrm{He}$ Yujin. Interactive Media Art. Chongqing: Southwest normal university press. 2008.

[6] Ascott, R. Technoetic Arts. Yonsei: Yonsei University Press. 2002.

[7] Woolner, A. Using interactive digital media to engage children on the autistic spectrum, A thesis submitted in partial fulfillment of the University's requirements for the Degree of Doctor of Philosophy. 2009.
[8] Cynthia Breazeal. Blended Reality Characters, Artificial Reality. ISBN 0-201-04765-9. 1. 2010.

[9] Wan Yan, Wan Rong \& Chen Shaohua. Multimedia Network Art. Chongqing: Southwest normal university press. 2007.

[10] Liu Shuming \& Hou Yue. Digital media interaction element design. Beijing: Higher Education Press, p3, p6. 2014.

[11] Jay David Bolter and Diane Gromala. Transparency and Reflectivity: Digital Art and the Aesthetics of Interface Design Chapter to appear in Fishwick, P. (ed.) Aesthetic computing. MIT Press. 2004.

[12] Myron Krueger. Artificial Reality. Addison-Wesley. ISBN 0-201-04765-9.1983.

[13] Stephen Wilson. Information Arts: Intersections of Art, Science, and Technology. Cambridge, Massachusetts: MIT Press. 2002.

[14] Wolf Lieser. The World of Digital Art.Publisher: H. F. Ullmann Publishing. ISBN-10: 3833153458. 2010. 